\title{
7 Zur Bedeutung körpermodifizierender Maßnahmen im Verlauf transsexueller Entwicklungen: ein Phänomen im Wandel
}

\author{
Timo 0. Nieder, Susanne Cerwenka und Hertha Richter-Appelt
}

In klinischen Zusammenhängen wird die häufig als selbstverständlich angenommene Verknüpfung von Identität und Körper in der Regel dann hinterfragt, wenn das eigene Geschlechtsidentitätserleben z.B. durch das Vorliegen von Unfruchtbarkeit verunsichert wird („Bin ich eine richtige Frau, ein richtiger Mann?"), wenn die geschlechtsspezifische und/oder -typische Ausprägung des Körpers nicht mit dem Geschlechtsidentitätserleben übereinstimmt (z.B. als Grundlage von transsexuellen Entwicklungen) oder wenn die Zuweisung einer Geschlechtsrolle bei Vorliegen eines nicht eindeutig männlichen oder weiblichen Körpers (z.B. bei intersexuellen Phänomenen) zur Diskussion steht. Gemein ist diesen Phänomenen die Abweichung von der Norm, dass Geschlechtsidentitätserleben, geschlechtsspezifische und -typische Körpermerkmale sowie die zugewiesene Geschlechtsrolle übereinstimmen.

Der vorliegende Beitrag stellt den Paradigmenwechsel im Hinblick auf die Behandlung der Geschlechtsdysphorie in den Mittelpunkt und beleuchtet die Bedeutung von körpermedizinischen Maßnahmen im Rahmen einer transsexuellen Entwicklung ergänzt um Daten aus dem Institut für Sexualforschung und Forensische Psychiatrie am Universitätsklinikum Hamburg-Eppendorf.

\subsection{Ausprägung und gängige Formen}

Im Rahmen von transsexuellen Entwicklungen erleben Männer und Frauen ihre Geschlechtsidentität nicht bzw. nicht vollständig im Einklang mit ihren geschlechtsbezogenen Körpermerkmalen und leben häufig keine eindeutig dem Körper entsprechende Geschlechtsrolle. Einige von ihnen streben eine 
7 Zur Bedeutung körpermodifizierender Maßnahmen im Verlauf transsexueller Entwicklungen: ein Phänomen im Wandel

körperliche Erscheinung an, die dem jeweiligen Geschlechtsidentitätserleben bzw. der gewünschten Geschlechtsrolle eher entspricht. Während unter dem Begriff „Körpermodifikationen“ häufig Veränderungen mit schmückendem, ästhetischem, dekorativem und künstlerischem Charakter subsumiert werden (z.B. Kasten 2006), liegt hingegen für Personen im Verlauf einer transsexuellen Entwicklung das Ziel der Behandlung in der Regel darin, dass ihre geschlechtsspezifischen Körpermerkmale mit ihrem Geschlechtsidentitätserleben übereinstimmen. Im Zuge einer zunehmenden Übereinstimmung von Geschlechtsidentitätserleben und geschlechtstypischer Erscheinung kann erreicht werden, dass die Beteiligten in ihrer gewünschten Geschlechtsrolle leben können und von ihrem jeweiligen Umfeld dem eigenen Geschlechtszugehörigkeitsempfinden entsprechend wahrgenommen werden.

Die Hintergründe, die zu einer transsexuellen Entwicklung führen können, sind äußerst heterogen und in der Regel ausschließlich individuell zu verstehen. In der Literatur besteht daher eine große Varianz im Hinblick auf Theorien zur Genese je nach Subgruppe und Perspektive. Typologien transsexueller Entwicklungen beziehen sich auf verschiedene Spezifizierungen, z.B. nach dem Herkunftsgeschlecht (Becker 2004), dem Alter bei Erstmanifestation (Nieder et al. 2011a) und der sexuellen Orientierung (Lawrence 2010).

\subsection{Häufigkeit}

Je nach Definition unterscheiden sich die Angaben zur Prävalenz. In Deutschland liegt die Anzahl transsexueller Frauen bei 5,5 auf 100.ooo (erfasst über die Entscheidungen zur Vornamens- und Personenstandsänderung; Meyer zu Hoberge 2009) und 7,8 bzw. 8,4 auf 100.ooo in Belgien und den Niederlanden (erfasst über genitalangleichende Operationen; Bakker et al. 1993; De Cuypere et al. 2007). Bei transsexuellen Männern liegen die Zahlen zwischen 3,1 auf 100.000 in Deutschland und 3 bzw. 3,3 in Belgien und den Niederlanden. Wird das Erleben von Geschlechtsdysphorie als Grundlage der Schätzung genutzt, steigen die Angaben zur Prävalenz auf bis zu 13,5 auf 100.00o bei anatomischmännlichen Personen (erfasst über Angaben der Hausärzte; Wilson et al. 1999).

\subsection{Kultur und Geschichte}

Die Indikationen für körpermedizinische Maßnahmen zur Veränderung der geschlechtsspezifischen und/oder geschlechtstypischen Erscheinung werden im Rahmen eines psychotherapeutischen Settings von spezialisierten psychologischen und ärztlichen Psychotherapeutinnen und Psychotherapeuten gestellt. Grundlegend für das bis dato vorherrschende Behandlungsparadigma der „Geschlechtsumwandlung“ (Pfäfflin u. Junge 1992) bzw. des „Geschlechtswechsel“ (Sigusch 1995) war die Metapher vom Leben „im falschen Körper“ (eine Frau gefangen im Körper eines Mannes respektive ein Mann gefangen 
im Körper einer Frau; vgl. u.a. Kamprad u. Schiffels 1991). Die Grundlage dafür bildete die konzeptionelle (und im englischen Sprach- und Kulturraum auch begriffliche) Trennung von sex und gender, die sich im Verlauf der zweiten Hälfte des 20. Jahrhunderts etablierte (Money 1994; Stoller 1964). Während der Begriff sex für gewöhnlich Merkmale des körperlichen Geschlechts erfasst, bezieht sich gender meist auf die psychosozialen und psychosexuellen Aspekte von Geschlecht. Gleichzeitig wurden durch die Separation der beiden Aspekte biologische Faktoren der Entwicklung von Geschlecht häufig monokausal der Kategorie sex zugeordnet und auf diese Weise die vielgestaltigen Wechselwirkungen zwischen Körper, Identität und Gesellschaft kaum berücksichtigt (vgl. Küppers 2012).

Betrachtet man den Wortlaut der Diagnose „Transsexualismus“ in der gegenwärtig gültigen und für die deutsche Ärzte- und Therapeutenschaft verbindlichen 10. Ausgabe der Internationalen statistischen Klassifikation der Krankheiten und verwandter Gesundheitsprobleme (ICD-10) fällt die Dreiteilung in ein Positivkriterium (1), ein Negativkriterium (2) und ein Behandlungskriterium (3) auf (Dilling et al. 2005):

1. „Es besteht der Wunsch, als Angehöriger des anderen anatomischen Geschlechtes zu leben und anerkannt zu werden."

2. „Dieser geht meist mit dem Gefühl des Unbehagens oder der Nichtzugehörigkeit zum eigenen Geschlecht einher."

3. „Es besteht der Wunsch nach hormoneller und chirurgischer Behandlung, um den eigenen Körper dem bevorzugten Geschlecht soweit wie möglich anzugleichen." (Dilling et al. 2005, 241)

Diese Verschränkung von Diagnose und Behandlung(-swunsch) stellt ein Unikum im Bereich F (Psychische und Verhaltensstörungen) der ICD-1o dar. Der Wunsch nach endokrinologischer und chirurgischer Veränderung der geschlechtsspezifischen Erscheinung des Körpers wird im Rahmen der diagnostischen Kriterien als obligat für die Diagnosestellung formuliert. Wiederholt wurde kritisiert (u.a. Becker 2009, 12), dass jene Menschen transsexuell seien, die „anhaltend und überzeugend ,geschlechtsumwandelnde ' Operationen“ anstreben. Neben dem Unbehagen mit den vorhandenen primären und sekundären Geschlechtsmerkmalen sowie dem Wunsch, in der Rolle des erlebten Geschlechts zu leben, galt der Wunsch bzw. das Bedürfnis nach körpermedizinischen Veränderungen der geschlechtsspezifischen Erscheinung als maßgebendes Kriterium.

\section{Was steckt hinter dieser Konstruktion?}

Im Lichte der erwähnten Metapher vom Leben im falschen Körper standen transsexuelle Personen insbesondere in der zweiten Hälfte des vergangenen Jahrhunderts (Meyerowitz 2002) unter der Forderung, den behandelnden Ärztinnen und Ärzten sowie Therapeutinnen und Therapeuten die Unvereinbarkeit ihres 
7 Zur Bedeutung körpermodifizierender Maßnahmen im Verlauf transsexueller Entwicklungen: ein Phänomen im Wandel

falschen Körpers mit dem innerem Erleben zu verdeutlichen. Um Zugang zu den körpermedizinischen Maßnahmen zur Veränderung der geschlechtsspezifischen Erscheinung zu bekommen, mussten sie den Eindruck hinterlassen, dass sie eigentlich das andere Geschlecht seien und schon immer waren (Nieder u. Richter-Appelt 2011). Um die Unveränderbarkeit und Unumkehrbarkeit ihrer transsexuellen Entwicklung zu demonstrieren, hatten transsexuelle Personen zunächst eine psychotherapeutische Behandlung zu absolvieren. Erst deren Scheitern bzw. das damit einhergehende Eingeständnis, dass allein mithilfe psychotherapeutischer Maßnahmen die Unvereinbarkeit von Geschlechtsidentitätserleben mit den geschlechtsspezifischen Körpermerkmalen nicht zu überwinden ist, legitimierte die körpermedizinischen Maßnahmen im Sinne einer ultima ratio (vgl. Burzig 1982; Pfäfflin 1994). Diese Herangehensweise gilt mittlerweile als grundlegend überholt. Insbesondere ist es nicht Aufgabe der Psychotherapie, zu prüfen, ob es sich um eine früher sogenannte wahre Transsexualität handelt (Becker 2006; Nieder 2010).

Ein weiterer Aspekt, dem eine grundlegende Bedeutung für die Diagnostik einer transsexuellen Entwicklung zugesprochen wurde (und zuweilen noch wird; vgl. Lawrence 2010), war die sexuelle Orientierung. Ab Mitte der 196oerJahre begannen Therapeutinnen und Therapeuten in den USA, ihre eigenen Kriterien zur Auswahl geeigneter Personen zur Geschlechtsangleichung heranzuziehen (Meyerowitz 2002). Ausschließlich jene transsexuellen Personen, die in Bezug auf ihr Geschlechtsidentitätserleben eine heterosexuelle Orientierung vorgaben, wurden als „echte Transsexuelle“ diagnostiziert (Becker 2006; Fisk 1973). Auch im Hinblick auf die jeweiligen Behandlungsmaßnahmen wurde der Weg als vorgezeichnet betrachtet: wenn schon transsexuell, dann äußerlich eindeutig als Frau bzw. eindeutig als Mann - Lösungswege mit einer uneindeutigen äußeren Erscheinung, die sich außerhalb der Binarität von Geschlecht (entweder Mann oder Frau) verorteten, waren nicht vorgesehen und nicht erwünscht (Nieder $u$. Richter-Appelt 2011). In der Folge galt daher auch für transsexuelle Männer und Frauen „die Regel der Unvereinbarkeit und Unveränderbarkeit: Jeder muss[te] jederzeit männlich oder weiblich sein." (Gildemeister 1992, S. 226).

Indem transsexuelle Menschen nach den Maßnahmen zur Veränderung der geschlechtsspezifischen Erscheinung exklusiv in den heterosexuellen Geschlechtsrollen von Mann und Frau lokalisiert wurden, konnte die heteronormative Grundlage zweigeschlechtlich organisierter Gesellschaftsstrukturen gewährleistet bleiben (vgl. Roen 2002). Wiederholt wurde von einem „Geschlechtscharakter" ausgegangen, der Merkmale auf der psychischen und Verhaltensebene enthält, die mit den geschlechtsspezifischen Körpermerkmalen korrespondieren (Hausen 1976, S. 363). Obgleich bereits in den 197oerJahren offensichtlich war, dass „Aussagen über den ,Geschlechtscharakter von Mann und Frau [...] normative Aussagen [seien] und als solche [...] in einem schwer zu erkennenden Verhältnis zur Realität" (Hausen 1976, S. 363) stünden, blieben in der klinischen Praxis stereotype Erwartungen an die Geschlechts- 
rollen von Mann und Frau sowohl auf Seite der Therapeuten und Therapeutinnen als auch auf Seite der transsexuellen Personen präsent (vgl. Meyerowitz 2002). Inwiefern der Zwang zur Eindeutigkeit die Unsicherheit der Behandelnden reflektierte oder direkt dem Erleben der Hilfe-Suchenden entsprach bzw. inwiefern die Eindeutigkeit des Geschlechtsidentitätserlebens in den Narrativen transsexueller Menschen iatrogen geformt wurde, bleibt in der Fachwelt umstritten (Nieder u. Richter-Appelt 2011; Pfäfflin 1997).

\subsection{Medizinpsychologischer Forschungsstand}

Vor dem skizzierten Hintergrund wurde in der bisherigen Geschichte des Phänomens Transsexualität wiederholt versucht, Unsicherheiten in der Behandlung von offensichtlich heterogenen transsexuellen Entwicklungswegen durch Kategorienbildung in Verlaufsformen und Typologien zu reduzieren (Becker 2004; Smith et al. 2005). Neben der getrennten Betrachtung der häufig als exklusiv aufgefassten zwei Geschlechter erfolgte die Bildung von Subgruppen in der Regel auf Basis zweier weiterer Faktoren:

- sexuelle Orientierung: Entwicklungen mit homosexueller Orientierung (bezogen auf das Geburtsgeschlecht) vs. Entwicklungen mit nicht-homosexueller Orientierung (u.a. Blanchard et al. 1987)

- Alter zum Zeitpunkt der Erstmanifestation (engl.: Age ofOnset): early-onset- vs. late-onset-Entwicklungen (vgl. Nieder et al. 2011a; Person u. Ovesey 1974a; Person und Ovesey 1974b).

Typologien werden in der klinischen Praxis häufig als rahmend und Halt gebend empfunden, zumal für jede Subgruppe unterschiedliche Annahmen zur Genese und Phänomenologie vorliegen. So lassen sich mithilfe von Typologien möglicherweise jene Irritationen versuchs- und ansatzweise ordnen, die im Kontakt mit uneindeutigen Erscheinungsformen im Zusammenhang mit transsexuellen Entwicklungen ausgelöst werden können. Andererseits kann das Vorhaben einer Typologisierung der Pluralität transsexueller Entwicklungen in der Realität kaum gerecht werden. Daher gewinnt die Nutzung multifaktorieller Heuristiken zunehmend an Bedeutung. In multifaktoriellen Heuristiken beeinflussen verschiedene neurobiologische Einflussfaktoren (genetische, endokrinologische, neurostrukturelle und -funktionelle Bedingungen; vgl. Nieder et al. 2011b) sowohl die somatosexuelle als auch die psychosexuelle Entwicklung. Hinweise liegen u.a. vor für das Geschlechtsidentitätserleben (u.a. Bao u. Swaab 2011), geschlechtsbezogene Verhaltensweisen (u.a. Hines 2010) und die sexuelle Orientierung (u.a. Sánchez et al. 2009). Wenn angenommen wird, dass die neurobiologischen Einflussfaktoren die fehlende Übereinstimmung von Verhalten, Körper und Erleben der beteiligten Person (mit-) bedingen, lässt sich folgern, dass die daraus resultierende (Geschlechts-)Inkongruenz zwischen Körper und Identität vermutlich in einem wechselseitigen Zusammenhang mit spezifischen Körper- und Beziehungserfahrungen 
7 Zur Bedeutung körpermodifizierender Maßnahmen im Verlauf transsexueller Entwicklungen: ein Phänomen im Wandel

(z.B. das Erleben von Symbiose bzw. Distinktion sowie Erfahrungen, begehrt zu werden; Richter-Appelt 2012) steht. Zudem wird der Einfluss soziologischer Bedingungen (z.B. kulturelle Paradigmen und soziale Praxen; u.a. Dietze u. Hark 2006) als prägnant aufgefasst. Im orchestralen Zusammenspiel der skizzierten Faktoren können die Grundlagen des Erlebens von Geschlechtsdysphorie gebildet werden. Der Begriff Geschlechtsdysphorie (engl.: gender dysphoria) bezeichnet den als krankheitswertig aufgefassten Leidensdruck, der sich infolge der skizzierten Inkongruenz entwickeln kann (Nieder u. Richter-Appelt 2012). Vor dem Hintergrund eines spezifischen kulturellen, psychosozialen und psychosexuellen Bedingungsgefüges kann das Erleben von Geschlechtsdysphorie im Verlauf der Ontogenese in eine transsexuelle Entwicklung münden, die ihrerseits mit körpermedizinischen Maßnahmen zur Veränderung der geschlechtsspezifischen und/oder -typischen Erscheinung einhergeht. Vor allem seit Beginn des neuen Jahrtausends vollzieht sich demnach schrittweise ein Paradigmenwechsel, innerhalb dessen die multimodale Gesundheitsfürsorge für Menschen mit einer transsexuellen Entwicklung in den Mittelpunkt rückt und die eingangs erwähnte Suche nach den „echten“ transsexuellen Entwicklungen an Bedeutung verliert.

Befunde aus Katamnesen und Verlaufsbeobachtungen zeigen, dass den körpermedizinischen Behandlungsmaßnahmen im Verlauf einer transsexuellen Entwicklung eine maßgebliche Bedeutung im Hinblick auf die Reduktion der Geschlechtsdysphorie und der Verbesserung des psychischen Wohlbefindens zukommt (im Überblick Murad et al. 2010; Wierckx et al. 2012). Dabei handelt es sich in der Regel um die Gabe von Sexualhormonen und deren Unterdrückung und chirurgische Veränderungen im Brust- und Genitalbereich. Bei transsexuellen Frauen (Personen mit weiblichem Geschlechtsidentitätserleben und geschlechtsspezifisch männlichen Körpermerkmalen), kommen die Epilationsbehandlung vor allem der Gesichtsbehaarung, häufig aber auch die chirurgische Verkleinerung des Kehlkopfes, seltener phonochirurgische Veränderungen sowie Maßnahmen zur chirurgischen Feminisierung des Gesichts hinzu. Jede Behandlungsmaßnahme sollte aus einer Verlaufsdiagnostik heraus individuell indiziert und psychotherapeutisch begleitet werden. Die Kombination von psychotherapeutischen, endokrinologischen und chirurgischen Behandlungsmaßnahmen ist geeignet, die individuelle Geschlechtsdysphorie nachhaltig zu reduzieren und die subjektive Zufriedenheit der Betreffenden mit ihrer Lebenssituation signifikant zu erhöhen (Gijs u. Brewaeys 2007; Löwenberg et al. 2011; Pfäfflin u. Junge 1990).

Im Rahmen einer multizentrischen, europäischen Vier-Länder-Studie zur Diagnostik und Behandlung der Geschlechtsdysphorie, an der sich am Institut für Sexualforschung und Forensische Psychiatrie am Universitätsklinikum Hamburg-Eppendorf eine Arbeitsgruppe beteiligt (Leitung: H. Richter-Appelt), konnte gezeigt werden, dass Personen mit einer transsexuellen Entwicklung von Frau zu Mann (im Folgenden Männer genannt) sich von solchen 
mit einer transsexuellen Entwicklung von Mann zu Frau (im Folgenden Frauen genannt) in der Verteilung der sexuellen Orientierungen sowie ihren Partnerschaftskonstellationen und partnerschaftlichen Ressourcen zum Zeitpunkt der Erstvorstellung unterscheiden (Cerwenka et al. 2012; Nieder et al. 2011a). Männer geben großteils eine sexuelle Orientierung auf Frauen sowie Partnerschaften mit Frauen an, die sich - entsprechend ihrem Identitätserleben - zu Männern hingezogen fühlen. Im Gegensatz dazu berichten Frauen deutlich häufiger von Partnerschaften, in denen die sexuelle Orientierung der (häufig weiblichen) Partner nicht zu ihrem Identitätsempfinden passt, sondern sich auf Männer (und damit auf ihre vorherige Geschlechtsrolle) bezieht. Für Frauen wurden zudem Unterschiede in den sexuellen Erlebens- und Verhaltensmustern gefunden zwischen Personen mit Beginn der transsexuellen Entwicklung vor der Pubertät (sogenannter early-onset) im Vergleich zur Manifestation während oder nach der Pubertät (sogenannter late-onset, Cerwenka et al. 2012). Frauen mit einer early-onset transsexuellen Entwicklung geben häufiger eine sexuelle Orientierung auf Männer an als Frauen mit einer late-onset transsexuellen Entwicklung. Ein größerer Anteil von Frauen mit einer early-onset transsexuellen Entwicklung berichtet überdies, in der partnerschaftlichen Sexualität die eigenen (körperspezifisch-männlichen) Genitalien nicht mit einzubeziehen sowie sexuelle Empfindungen am Penis und den Orgasmus als eher unangenehm zu erleben.

\subsection{Umgang im medizinischen Altag}

Von dem skizzierten Konzept zur Etablierung der Geschlechtsdysphorie als Behandlungsfokus lassen sich im Rahmen persönlicher psychodiagnostischer Gespräche Annahmen ableiten, auf welchen Bedingungen im Einzelfall die Geschlechtsinkongruenz basiert sowie aus welchen Faktoren sich das individuelle Erleben von Geschlechtsdysphorie speist: So sind Manche vorwiegend belastet durch die fehlende Anerkennung ihrer Identität bzw. durch die fehlende Akzeptanz ihres Verhaltens, während Andere maßgeblich unter dem Ekel bzw. der Abscheu vor ihren (insbesondere sekundären) geschlechtsspezifischen Körpermerkmalen leiden. Wichtig ist daher, konkrete Überlegungen anzustellen, welche körpercharakteristischen Veränderungen (z.B. Art der Körperund Gesichtsbehaarung, Umverteilung von Körperfett und Muskelmasse, Aufbau und Gestaltung des Brustprofils, Ausstattung des Genitalbereichs, Stimmhöhe etc.) mit welcher Behandlungsmaßnahme (Gabe von Sexualhormonen und deren Suppression, Epilation, chirurgische Veränderungen des Brustprofils und des Genitalbereichs, chirurgische Verkleinerung des Kehlkopfes und logopädische sowie phonochirurgische Veränderungen von Stimmbild und -höhe) in der Lage sind, zu einer signifikanten und dauerhaften Reduktion der Geschlechtsdysphorie beizutragen. Der Körper bzw. das Körpererleben spielt in der Behandlung der Geschlechtsdysphorie folgerichtig eine zentrale Rolle. 
7 Zur Bedeutung körpermodifizierender Maßnahmen im Verlauf transsexueller Entwicklungen: ein Phänomen im Wandel

Der skizzierte Paradigmenwechsel in der Behandlung wird von der jüngst veröffentlichten 7. Version der internationalen Standards of Care (SoC 7) der World Professional Association of Transgender Health (WPATH) reflektiert (Coleman et al. 2011). Aus Katamnesen und klinischen Studien liegt den endokrinologischen und chirurgischen Behandlungsmaßnahmen Evidenz zur Reduktion der Geschlechtsdysphorie und einer Verbesserung der Lebensqualität zugrunde, wenngleich die Datenlage aus methodischen Gesichtspunkten als spärlich zu betrachten ist (Byne et al. 2012; Murad et al. 2010; Sutcliffe et al. 2009). Die Psychotherapie kann dabei sowohl die endokrinologischen und/oder chirurgischen Maßnahmen ergänzen sowie als eigenständige Maßnahme zur Linderung des Leidens unter der Geschlechtsdysphorie indiziert sein. Aus der Verlaufsdiagnostik heraus bzw. im Rahmen einer psychotherapeutischen Begleittherapie (die sich der Verlaufsdiagnostik anschließen und z.B. an einer psychiatrischen Institutsambulanz mit sexualtherapeutischem Schwerpunkt durchgeführt werden kann) können verschiedene somatomedizinische Maßnahmen zur Behandlung der Geschlechtsdysphorie bzw. zur Veränderung der geschlechtsspezifischen Erscheinung indiziert werden.

Zur Vorbereitung auf die Indikationsstellungen möglicher somatomedizinischer Maßnahmen im Verlauf einer transsexuellen Entwicklung ist es von zentraler Bedeutung, Überlegungen anzustellen, aus welchen Faktoren sich die Geschlechtsdysphorie im Einzelnen speist. Auf dieser Grundlage lässt sich am ehesten die Frage beantworten, welche körpercharakteristischen Veränderungen (z.B. Aufbau und Gestaltung eines passenden Brustprofils) infolge welcher Behandlungsmaßnahmen (z.B. chirurgische Veränderungen des Brustprofils) eine signifikante und dauerhafte Reduktion der Geschlechtsdysphorie erreicht werden kann. Die nachhaltige Reduktion des Erlebens von Geschlechtsdysphorie ist dabei das oberste Ziel der Behandlung. Innerhalb der psychotherapeutischen Arbeit sind in diesem Zusammenhang die bewusst oder unbewusst angewandten Wertungen des jeweils individuellen Geschlechtsentwurfes transsexueller Menschen kritisch zu reflektieren. Im Rahmen der Behandlung der Geschlechtsdysphorie ist es daher ratsam, mit individuellen Identitätsbildungen achtsam umzugehen und bei Bedarf möglichen Entwicklungen außerhalb der Binarität von Geschlecht Raum zu geben.

\subsection{Abschließende Überlegungen zur Bedeutung körpermodifizierender Maßnahmen im Verlauf transsexueller Entwicklungen: notwendige, nicht hinreichende Bedingungen zur Reduktion der Geschlechtsdysphorie}

Der zentrale Stellenwert, den körpermodifizierende Maßnahmen in der Wahrnehmung jener Menschen einnehmen, die aufgrund ihres Geschlechtsdysphorieerlebens professionelle Hilfe suchen, lässt sich anhand einiger Zahlen verdeutlichen. Im Zeitraum vom Januar 2007 bis September 2011 wandten sich 
103 Personen mit transsexueller Entwicklung an die Psychiatrische Institutsambulanz am Institut für Sexualforschung und Forensische Psychiatrie des Universitätsklinikums Hamburg-Eppendorf (UKE). 49 von ihnen erlebten sich bei männlichen Körpermerkmalen vorwiegend weiblich (d.h. Frauen), 54 Personen erleben sich bei weiblichen Körpermerkmalen vorwiegend männlich (d.h. Männer). Das Verhältnis zwischen den Geschlechtern lag bei 1:1,10.

In der vorliegenden Stichprobe waren die Frauen (Alter $M d=33$, Range 1858 Jahre) zum Zeitpunkt der Erstvorstellung durchschnittlich älter als die Männer (Alter $M d=24$, Range 16-6o Jahre; $\mathrm{U}=753.50, p<0.001$ ). In beiden Geschlechtergruppen gab ein hoher Prozentsatz der Personen an, sich allgemein körpermedizinische Behandlungen zur Veränderung der geschlechtsspezifischen Körpermerkmale zu wünschen (Frauen: 89,8\%; $n=44$, Männer: 83,3\%, $\mathrm{n}=45$ ).

Obgleich sich jeweils ein größerer Anteil von Personen sowohl endokrinologische als auch chirurgische Maßnahmen wünschte (Frauen: 72,7\%, n = 32; Männer: $65,9 \%, \mathrm{n}=29$ ), deuteten sich Geschlechterunterschiede in der Gewichtung der gewünschten Veränderungen der geschlechtsspezifischen Erscheinung an. So strebten Frauen vergleichsweise häufiger ausschließlich eine Behandlung mit Sexualhormonen und deren Suppression an (Frauen: $18,2 \%, n=8$; Männer: $11,4 \%, n=5$ ), während für einen höheren Anteil von Männern ausschließlich chirurgische Maßnahmen im Vordergrund zu stehen schienen (Frauen: 9,1\%, n = 4; Männer: 22,7\%, n = 10). Maßnahmen zur Veränderung der körperlichen Erscheinung im Rahmen einer transsexuellen Entwicklung scheinen daher in der Wahrnehmung der Betreffenden aus der vorliegenden Stichprobe zumindest zu Beginn der Behandlung einen zentralen Stellenwert einzunehmen. Den Daten aus der systematischen Evidenzbasierung, nach denen die genitalchirurgischen Maßnahmen bei den Frauen sowohl im Hinblick auf post-operative Funktionalität und Sensitivität als auch im Hinblick auf eine geringere Komplikationsrate zu besseren Outcomes bei den Frauen führen (vgl. Sutcliffe et al. 2009), entspricht auch die klinische Erfahrung insofern, dass Frauen genitalchirurgische Maßnahmen im weiteren Verlauf transsexueller Entwicklungen häufiger als Männer durchführen lassen.

Die enge Verschränkung zwischen Körper- und Identitätserleben, zwischen Geschlechtsidentitätsempfinden und der Zuordnung zu einem Geschlecht auf Basis der körperlichen Erscheinung durch Andere scheint spezifisch für transsexuelle Entwicklungen. Menschen mit einem Geschlechtszugehörigkeitsempfinden, das sich in diesem Sinne nicht eindeutig innerhalb der zweigeschlechtlich organisierten westlichen Gesellschaften kategorisieren lässt, stehen daher häufig unter einer großen Belastung. Der psychische Leidensdruck, der sich daraus ergeben kann, wurde für die Lebenssituation transsexueller Menschen ausführlich beschrieben (Meyer-Bahlburg 2010). Körpermodifizierende Maßnahmen tragen entscheidend zur Erhöhung der subjektiven Lebenszufriedenheit und der Lebensqualität der Betreffenden (Gijs u. Brewaeys 2007; 
Pfäfflin u. Junge 1992), und damit zur nachhaltigen Reduktion der Geschlechtsdysphorie im Rahmen einer individuellen Behandlung, bei.

Inwiefern der skizzierte Paradigmenwechsel hin zu einer effizienten und multimodalen Therapie zur Etablierung eines nachhaltigen Wohlbefindens im Kontext individueller (Trans-)Identität auch in einen Zusammenhang mit der Zunahme von Körpermodifikationen insgesamt in der Gesellschaft zu stellen ist (vgl. Borkenhagen u. Brähler 2010), lässt sich im Rahmen des vorliegenden Beitrages nicht abschließend klären. Zudem fehlen neuere katamnestische Erhebungen, die den Paradigmenwechsel in der Behandlung der Geschlechtsdysphorie empirisch fassen. Körpermodifikationen, die nicht in ein vergleichbares Paradigma eingebettet sind und für die keine Daten aus Katamnesen zum „Behandlungserfolg“ der jeweiligen Maßnahme vorliegen, lassen sich nur schwer mit den körpermedizinischen Behandlungsmaßnahmen im Verlauf einer transsexuellen Entwicklung vergleichen. Insofern sind die somatomedizinischen Veränderungen der geschlechtsspezifischen Erscheinung zur Behandlung einer ausgeprägten und persistierenden Geschlechtsdysphorie trotz ihres offensichtlich körpermodifizierenden Charakters nicht der bisherigen Definition von Körpermodifikationen zuzuordnen.

\section{Literatur}

Bakker A, van Kesteren PJ, Gooren LI, Bezemer PD (1993) The prevalence of transsexualism in The Netherlands. Acta Psychiatr Scand 87, 237-238

Bao A-M, Swaab DF (2011) Sexual differentiation of the human brain: Relation to gender identity, sexual orientation and neuropsychiatric disorders. Frontiers in neuroendocrinology 32, 214-226

Becker S (2004) Transsexualität - Geschlechtsidentitätsstörung. In: Kockott G, Fahrner E-M (Hrsg.) Sexualstörungen. 153-201. Thieme Stuttgart

Becker S (2006) Abschied vom „echten“ Transsexuellen. Zeitschrift für Sexualforschung 19, 154-158

Becker S (2009) Transsexuelle Entwicklungen: Verlaufsdiagnostik, Psychotherapie und Indikation zu somatischen Behandlungen Psychotherapie im Dialog 10, 12-18

Blanchard R, Clemmensen LH, Steiner BW (1987) Heterosexual and homosexual gender dysphoria. Archives of sexual behavior 16, 139-152

Borkenhagen A, Brähler E (Hrsg.) (2010) Intimmodifikationen. Spielarten und ihre psychosozialen Bedeutungen. Psychosozial-Verlag Gießen

Burzig G (1982) Der Psychoanalytiker und der transsexuelle Patient. Ein Beitrag zur notwendigen Auseinandersetzung mit „psycho“-chirurgischen Eingriffen an den Geschlechtsmerkmalen. Psyche 36, 846-856

Byne W, Bradley SI, Coleman E, Eyler AE, Green R, Menvielle E), Meyer-Bahlburg HF, Pleak RR, Tompkins DA. Report of the American Psychiatric Association task force on treatment of Gender Identity Disorder. Archives of sexual behavior 2012; 41: 759-796

Cerwenka S, Nieder TO, Richter-Appelt H (2012) Sexuelle Orientierung und Partnerwahl transsexueller Frauen und Männer vor körpermedizinischen geschlechtsanpassenden Maßnahmen. Psychother Psych Med 62, 214-222

Coleman E, Bockting W, Botzer M, Cohen-Kettenis P, DeCuypere G, Feldman I, Fraser L, Green I, Knudson G, Meyer W, Monstrey S, Adler R, Brown G, Devor A, Ehrbar R, Ettner R, Eyler E, Garofalo R, Karasic D, Lev Al, Mayer G, Meyer-Bahlburg H, Hall BP, Pfäfflin F, Rachlin K, Robinson B, Schechter L, Tangpricha V, van Trotsenburg M, Vitale A, Winter S, Whittle S, Wylie K, Zucker K (2011) Standards of Care for the Health of Transsexual, Transgender, and Gender Nonconforming People. 7th Version. WPATH Minneapolis 
De Cuypere G, Van Hemelrijck M, Michel A, Carael B, Heylens G, Rubens R, Hoebeke P, Monstrey S (2007) Prevalence and demography of transsexualism in Belgium. Eur Psychiatry 22, 137-141

Dietze G, Hark S (Hrsg.) (2006) Gender kontrovers - Genealogien und Grenzen einer Kategorie. Ulrike Helmer Verlag Königstein/Taunus

Dilling H, Mombour W, Schmidt MH (2005) Internationale Klassifikation psychischer Störungen, Kapitel V (F). Hans Huber Bern

Fisk N (1973) Gender Dysphoria Syndrome (The how, what, and why of a disease). In: Laub D, Gandy P (Hrsg.) Proceedings of the 2nd Interdisciplinary Symposium on Gender Dysphoria Syndrome. 7-14. University of California Press Stanford

Gijs L, Brewaeys A (2007) Surgical Treatment of Gender Dysphoria in Adults and Adolescents: Recent Developments, Effectiveness, and Challenges. Annual Review of Sex Research 18, 178-224

Gildemeister R (1992) Die soziale Konstruktion von Geschlechtlichkeit. In: Ostner I, Lichtblau K (Hrsg.) Feministische Vernunftkritik Ansätze und Traditionen. 220-239. Campus Frankfurt a.M.

Hausen K (1976) Die Polarisierung der „Geschlechtscharaktere“. Eine Spiegelung der Dissoziation von Erwerbsund Familienleben. In: Conze W (Hrsg.) Sozialgeschichte der Familie in der Neuzeit Europas. 363-393. Klett-Cotta Stuttgart

Hines M (2010) Sex-related variation in human behavior and the brain. Trends Cogn Sci 14, 448-456

Kamprad B, Schiffels W (Hrsg.) (1991) Im Falschen Körper - Alles über Transsexualität. Kreuz Verlag Zürich

Kasten E (2006) Body-Modification. Psychologische und medizinische Aspekte von Piercing, Tattoo, Selbstverletzung und anderen Körperveränderungen. Ernst Reinhardt Verlag München

Küppers C (2012) Soziologische Dimensionen von Geschlecht. Aus Politik und Zeitgeschichte 20-21, 3-8

Lawrence AA (2010) Sexual Orientation versus Age of Onset as Bases for Typologies (Subtypes) for Gender Identity Disorder in Adolescents and Adults. Archives of sexual behavior 39, 514-545

Löwenberg H, Lax H, Rossi Neto R, Krege S (2011) Komplikationen, subjektive Zufriedenheit und sexuelles Erleben nach geschlechtsangleichender Operation bei Mann-zu-Frau-Transsexualität. Z Sexualforsch 23, 328-347

Meyer zu Hoberge S (2009) Prävalenz, Inzidenz und Geschlechterverhältnis der Transsexualität anhand der bundesweit getroffenen Entscheidungen nach dem Transsexuellengesetz in der Zeit von 1991 bis 2000. Sektion für Sexualmedizin. Christian-Albrechts-Universität zu Kiel, Dissertation

Meyer-Bahlburg H (2010) From mental disorder to iatrogenic hypogonadism: Dilemmas in conceptualizing gender identity variants as psychiatric conditions. Archives of sexual behavior 39, 461-476

Meyerowitz I (2002) How Sex Changed - A History of Transsexuality in the United States. Harvard University Press Cambridge

Money I (1994) Zur Geschichte des Konzepts Gender Identity Disorder. Zeitschrift für Sexualforschung 7, 20-34

Murad MH, Elamin MB, Garcia MZ, Mullan RJ, Murad A, Erwin PJ, Montori VM (2010) Hormonal therapy and sex reassignment: A systematic review and meta-analysis of quality of life and psychosocial outcomes. Clin Endocrinol 72(2), 214-231

Nieder T0 (2010) Transsexuelle Entwicklungen und therapeutische Praxis. Zeitschrift für Sexualforschung 23, 63-70

Nieder TO, Herff M, Cerwenka S, Preuss WF, Cohen-Kettenis PT, De Cuypere G, Haraldsen IR, Richter-Appelt H (2011a) Age of onset and sexual orientation in transsexual males and females. I Sex Med 8, 783-791

Nieder TO, Jordan K, Richter-Appelt H (2011b) Zur Neurobiologie transsexueller Entwicklungen - Eine Diskussion der Befunde zur Sexualdifferenzierung, geschlechtsatypischen Verhaltensweisen und Geschlechtsidentität. Zeitschrift für Sexualforschung 24, 199-227

Nieder TO, Richter-Appelt H (2011) Tertium non datur - either/or reactions to transsexualism amongst health care professionals: the situation past and present, and its relevance to the future. Psychology \& Sexuality 2, 224-243

Nieder T0, Richter-Appelt H (2012) Transsexualität und Geschlechtsdysphorie. Gynäkologie Geburtsmedizin Gynäkologische Endokrinologie 8, 60-71

Person E, Ovesey L (1974a) The transsexual syndrome in males: I. Primary transsexualism. American Journal of Psychotherapy 28, 4-20 


\section{Zur Bedeutung körpermodifizierender Maßnahmen im Verlauf}

transsexueller Entwicklungen: ein Phänomen im Wandel

Person E, Ovesey L (1974b) The transsexual syndrome in males: II. Secondary transsexualism. American Journal of Psychotherapy 28, 174-193

Pfäfflin F (1994) Zur transsexuellen Abwehr. Psyche 48, 904-931

Pfäfflin F (1997) Das Problem der latrogenese am Beispiel der Transsexualität. In: Willenberg H, Hoffmann SO (Hrsg.) Handeln - Ausdrucksform psychosomatischer Krankheit und Faktor der Therapie. 173-178. VAS Verlag für akademische Schriften Frankfurt a.M.

Pfäfflin F, Junge A (1990) Nachuntersuchung von 85 operierten Transsexuellen. Zeitschrift für Sexualforschung 3, 331-348

Pfäfflin F, Junge A (Hrsg.) (1992) Geschlechtsumwandlung: Abhandlungen zur Transsexualität. Schattauer Stuttgart

Richter-Appelt H (2012) Geschlechtsidentität und -dysphorie aus psychoanalytischer Sicht. Aus Politik und Zeitgeschichte 20-21, 22-28

Roen K (2002) "Either/Or" and "Both/Neither": Discursive Tensions in Transgender Politics. Signs 27, 501-522

Sánchez FI, Bocklandt S, Vilain E (2009) The Biology of Sexual Orientation and Gender Identity. In: Pfaff DW, Arnold AP, Etgen AM, Fahrbach SE, Rubin RT (Hrsg.) Hormones, Brain and Behavior. 1911-1929. Academic Press New York

Sigusch V (1995) Geschlechtswechsel. Rotbuch-Verlag Hamburg

Smith YL, Van Goozen SH, Kuiper AI, Cohen-Kettenis PT (2005) Transsexual subtypes: clinical and theoretical significance. Psychiatry Res 137, 151-160

Stoller RJ (1964) A Contribution to the Study of Gender Identity. The International journal of psycho-analysis $45,220-226$

Sutcliffe PA, Dixon S, Akehurst RL, Wilkinson A, Shippam A, White S, Richards R, Caddy CM (2009) Evaluation of surgical procedures for sex reassignment: a systematic review. J Plast Reconstr Aesthet Surg 62, 294-306; discussion 306-298

Wierckx K, Mueller S, Weyers S, Van Caenegem E, Roef G, Heylens G, T'Sjoen G (2012) Long-Term Evaluation of Cross-Sex Hormone Treatment in Transsexual Persons. The journal of sexual medicine 9(10), 2641-2651

Wilson P, Sharp C, Carr S (1999) The prevalence of gender dysphoria in Scotland: a primary care study. Br I Gen Pract 49, 991-992 